\title{
¿Es posible que nuestros/as estudiantes adquieran la habilidad de comunicarse con fluidez en su entorno y de trabajar en equipo?
}

\author{
Ana Cano-Ramírez ${ }^{\text {a }}$, Francisco S. Cabrera-Suárez ${ }^{\text {b }}$
}

${ }^{a}$ Universidad de Las Palmas de Gran Canaria, Las Palmas, España, ana.cano@upgc.es, b a Universidad de Las Palmas de Gran Canaria, Las Palmas, España, fcabrera@dma.upgc.es

\begin{abstract}
Resumen
Nos proponemos con este trabajo analizar si la experiencia metodológica realizada con nuestros/as estudiantes de Grado de Trabajo Social les permite avanzar en la adquisición de una de las competencias generales que se contemplan en los proyectos docentes de la asignatura Programas y Prestaciones de los Servicios Sociales ( $2^{\circ}$ curso) de Grado de Trabajo Social de la Universidad de Las Palmas de Gran Canaria (ULPGC). Dicha competencia está definida como "CG2. Ser capaz de comunicarse con fluidez en su entorno y trabajar en equipo."

Para ello entendemos que el atributo definido por Boni et. al. (2013) como "capacidad de comunicar y colaborar con otros", siendo una de las habilidades que participa de la perspectiva pedagógica basada en la Educación para el Desarrollo para la Ciudadanía Global, está directamente relacionada con la competencia general de la asignatura señalada anteriormente.
\end{abstract}

Por la dificultad que entraña realizar una evaluación directa por el profesorado sobre los avances de aprendizaje de esta habilidad, hemos acudido a un cuestionario como herramienta de acceso a la opinión que los/as propios/as estudiantes realizan sobre si la experiencia docente les ha procurado avanzar en este aprendizaje.

La metodología docente aplicada se apoya en el Aprendizaje-Servicio, y los datos obtenidos se corresponde con la acumulación de la experiencia de los dos últimos años de docencia.

Palabras clave: Educación para el Desarrollo para la Ciudadanía Global, habilidad, Aprendizaje-Servicio. 
¿Es posible que nuestros/as estudiantes adquieran la habilidad de comunicarse con fluidez en su entorno y de trabajar en equipo?

\section{Introducción}

El trabajo que se presenta en las estas páginas, se inspira en la búsqueda de ordenar y sistematizar la práctica docente a la par que dotarla de coherencia y consistencia. Se plantea como un ejercicio de reflexión sobre la propia tarea docente, a fín de contrastar y verificar el sentido y significado que la misma adquiere a través de una doble mirada que se complementa, y que se refleja en la finalidad que se persigue.

Así pues, la finalidad de este trabajo contempla esa doble vertiente:

- de un lado, revisar del currículum institucional para determinar la consistencia de seguir trabajando en la adquisición de una de las competencias generales que se contemplan en el proyecto docente, definida como "Competencia General 2. Ser capaz de comunicarse con fluidez en su entorno y trabajar en equipo.".

- de otro lado, analizar en qué medida la experiencia pedagógica realizada con nuestros/as estudiantes les permite avanzar en la adquisición de dicha competencia.

Este esfuerzo de análisis reflexivo de la actividad docente viene fundamentada por tres acontecimientos que han venido emergiendo en los últimos años, los cuales convergen en interpelar al sistema universitario un papel más activo en la consecución de un modelo educativo que se adecúe al contexto actual.

Estos tres acontecimientos son: uno, la creación de un Espacio Europeo de Educación Superior, que promueve la innovación docente como estrategia que impulsa y promueve la incorporación de mecanismos docentes distintos y alternativos al modelo educativo tradicional; dos, la reflexión que cada vez se hace más extensa sobre el papel que la Responsabildad Social Universitaria, invitando a una revisión sobre cómo y sobre qué criterios la institución aborda sus misiones (investigación, docencia, gobernanza y proyección social); y, tres, la más reciente Declaración de los Objetivos de Desarrollo Sostenible por Naciones Unidas, que interpela a todos los agentes (incluida a la Universidad) a asumir su compromiso y su papel activo en la consecución de los diecisiete objetivos planteados a alcanzar en el año 2030.

En este marco, la actividad docente está atrapada en una revisión reflexiva constante. He aquí un esfuerzo por rendir cuenta (en ese ejercicio de responsabilidad social), para estudiar si efectivamente el esfuerzo que se hace (en este caso en una asignatura concreta) está alineada con las condiciones y exigencias a las que ha de dar cumplimiento.

Para ello, y concretando el foco de atención, dentro del conjunto de competencias que se viene trabajando con los/as estudiantes, es la habilidad de comunicarse con fluidez en su entorno y de trabajar en equipo, sobre la que se desarrolla la actividad de análisis y reflexión. 
En este sentido, se ordena los siguientes apartados atendiendo, en primer lugar, a presentar la experiencia docente, el modelo educativo en el que se inserta y las esrategias metodológicas que la integran; en segundo lugar, se presenta los resultados obtenidos, por un lado, de la revisión de la literatura que construye el currículum institucional sobre la competencia que centra la atención y, por otro lado, los resultados de las voces de los/as estudiantes sobre la consideración que ellos/as tienen respecto a la adquisición de la competencia atenciendo a cada estrategia metodológica. En tercer lugar, se sigue con el apartado de análisis de los resultados, para finalizar, en cuarto lugar, con las conclusiones que se alcanzan.

\section{La experiencia docente}

La asignatura Programas y Prestaciones de los Servicios Sociales se inserta en el primer semestre del segundo curso del Grado de Trabajo Social, en la ULPGC. Se trata de un único grupo de estudiantes (85 matriculados/as en 2016-17; 96 en el curso 2017-18).

Las estrategias metodológicas que vienen impuestas por el diseño del título son: las clases de gran grupo, las clases prácticas (se distribuye el alumnado en dos grupos), el trabajo grupal.

La baremación de la calificación es de 60\% (examen sobre las nociones teóricas), 30\% (trabajo grupal), y 10\% asistencia y participación.

La estrategia pedagógica de las clases del gran grupo, se distribuye en dos enfoques atendiendo al porcentaje de horas que designa el Titulo de Grado en Trabajo Social (ULPGC, 2010):

- Las clases de gran grupo que atiende a los contenidos conceptuales: dado el ratio, los tiempos (dos sesiones de clases, de dos horas cada una) y los espacios físicos, obligan a un modelo educativo tradicional, con un predominio de clases magistrales basada en la teoría de aprendizaje conductista, e intercaladas con algunas clases apoyadas en dinámicas grupales, cuyo objetivos persigue, fundamentalmente, reforzar nociones conceptuales y generar estímulos para captar la atención del alumnado.

- Las clases de gran grupo que tiene asignada el trabajo grupal: se realiza un diseño de trabajo grupal que combina el esfuerzo presencial y no presencial. Las horas presenciales se distribuyen en: tutorías grupales, que tienen como objetivo acompañar a cada grupo para atender dudas, orientar, canalizar cada grupo; asambleas de gran 
¿Es posible que nuestros/as estudiantes adquieran la habilidad de comunicarse con fluidez en su entorno y de trabajar en equipo?

grupo: negociar, dialogar, compartir y decidir el tipo de trabajo que como grupo clase configurarán en conjunto todos los grupos; y sesiones de trabajo de los grupos, se configuran en horas de encuentro para que los grupos avancen en sus trabajos.

La estrategia de trabajo impulsada en esta parte de la asignatura se aborda desde la innovación educativa, ofreciendo a los/as estudiantes la posibilidad de generar un formato de presentación de los resultados de sus respectivos trabajos atendiendo a criterios de utilidad y significado.

Estos dos años de experiencia se ha asumido que la presentación de los resultados de los trabajos grupales, salen del aula para ofrecerlos a la comunidad universitaria, celebrándose sendos Encuentros de Sensibilización sobre Realidades Sociales en la Facultad de Economía, Empresa y Turismo y la Facultad de Ciencias Juridicas (201617), y en la Escuela de Ingeniería de Telecomunicación y Electrónica y en la Escuela de Ingenierías Industriales y Civiles (2017-18).

El diseño del trabajo grupal se centra en el enfoque educativo de la Educación para el Desarrollo para la Ciudadanía Global (EDCG) (Ortega Carpio, 2007; Cano, 2014), tomando el Aprendizaje-Servicio (ApS) (Aramburuzabala, 2013:6) como herramienta práctica para desarrollar la tarea docente. Esta estrategia metodológica se nutre de las teorías constructivistas de la psicología y de la las teorías contemporáneas de la pedagogía. Entre ellas, se desprende con especial protagonismo las siguientes: Pedagogía de la Liberación de Paulo Freire, la Teoría de Construcción Histórico-sociocultural del Conocimiento de Vigotsky, la Teoría del Aprendizaje Significativo de Ausubel y la Teoría de los Intereses del Conocimiento de Habermas (Díaz, 2010), teorías, todas ellas, inspiradoras de la iniciativa pedagógica que se lleva a cabo.

- Las clases prácticas, al reducirse el ratio de alumnado a la mitad, favorece la realización de actividades más activas y dinámicas (cada grupo 1 sesión de 55 minutos a la semana).

\section{Resultados}

Los datos obtenidos de la práctica proceden de una encuesta que los/as estudiantes cumplimentan una vez finaliza la experiencia académica. Los resultados de la literatura se nutre del currículum académico (Libro Blanco de Trabajo Social -2004- y del Plan del Título de Trabajo Social -2010- de la ULPGC) y del curróculum profesional (Código Deontológico del Consejo General de Trabajo Social (2012). 


\section{1. ¿Qué dice el currículum institucional sobre la habilidad de comunicarse con fluidez en su entorno y de trabajar en equipo? Resultados}

Con relación a la competencia general de la asignatura Programas y Prestaciones de los Servicios Sociales ser capaz de comunicarse con fluidez en su entorno y trabajar en equipo, se identifica en el Libro Blanco de Trabajo Social (2004) las siguientes competencias que ha de adquirir/desarrollar un/a Trabajador/a Social: comunicación oral y escrita en la lengua nativa (competencia transversal), trabajo en equipo y el trabajo en equipo de carácter interdisciplinar (competencias personales).

En el Título de Grado de Trabajo Social de la ULPGC la citada competencia aparece en el Módulo A. El Trabajo Social: conceptos, métodos, teorías y aplicación; Módulo B: El contexto institucional del Trabajo Social (en el que se inserta la asignatura); Módulo D: Herramientas legales, idiomáticas y organizativas para el Trabajo Social; y Módulo E: Prácticas y Trabajo fin de Grado.

Para finalizar esta sección, se presenta aspectos del Código Deontológico del Trabajo Social en los que se requiere contar con el desarrollo de esta competencia. En el Código se señala que los/as trabajadores/as sociales "deben comunicar o denunciar las situaciones de maltrato, abuso o abandono de las personas indefensas o incapacitadas, manteniendo no obstante el objetivo de intervenir para corregir esas situaciones con las personas implicadas en la misma. Igualmente debe denunciar cualquier otra situación que vaya contra los derechos humanos" (Artículo 23). A su vez, cuando "se encuentre en situación de sufrir una perturbación por el mantenimiento del secreto profesional, debe comunicarlo por escrito a la Junta de Gobierno de su Colegio Oficial para obtener la defensa y protección colegial en el uso del secreto dentro de su actuación profesional (Artículo 41). Con relación al trabajo en equipo "tiene la responsabilidad de evaluar su trabajo y el de su equipo de manera leal y respetuosa, de acuerdo con criterios objetivos" (Artículo 53).

\section{2. ¿Qué dicen los/as estudiantes sobre la adquisición de la habilidad como resultado de la experiencia docente?}

Llevada a la práctica docente la iniciativa, se hace necesario analizar y reflexionar sobre aquellos resultados que se han estado acopiando y revertir la mirada sobre la narrativa del currículum institucional para verificar si el currículum del diseño de la titulación se refleja en la praxis docente. 
Los datos que se disponen proceden de una encuesta de respuesta dicotómica (sí/no), que los/as estudiantes responden de manera voluntaria y anónima. Esta encuesta contempla los atributos conceptuales, de habilidades y actitudinales propuestos por Boni et.al. (2013).

Dado que el planteamiento, desde que se presenta el proyecto docente al inicio del semestre, es que la asignatura se constituye en un proyecto que cuenta en su haber unos objetivos, unas herramientas metodológicas, unos recursos (dícese recursos humanos: estudiantes y profesora; recursos de infraestructura: aula, entorno; recurso tiempo: horas de clases presenciales, horas de clase no presenciales; una calendarización impuesta por la institución: plazos de trabajo, exámenes; y una finalidad a alcanzar: que aprendan y superen la asignatura), la última sesión de clase presencial queda reservada para que estudiantes y profesora realicemos una valoración global sobre el cómo y lo que hemos trabajado.

Parte de esta valoración es cumplimentar la encuesta con la finalidad de obtener un feedback respecto a la percepción que ellos/as tienen sobre lo que el proyecto-asignatura les ha permitido desarrollar los atributos que se identifican en la misma.

Dicha encuesta se estructura con base a las tres estrategias medotológicas que se desarrollan a lo largo del semestre, y que han sido citadas anteriormente: las clases con gran grupo, en la que se combina las explicaciones de las nociones a través de clases magistrales, fundamentalmente, con algunas clases más dinámicas; el trabajo grupal obligatorio y las clases prácticas.

Se ha logrado un total de 117 encuestas (52 del curso 2016-17, y 65 del curso 2017-18). De ellas se ha obtenido los siguientes resultados atendiendo a cada una de las estrategias metodológicas:

a) Con relación a las clases teóricas del gran grupo, se obtiene que del total de encuestas respondidas en el curso 2016-17, sólo 10 estudiantes entienden que esta estrategia metodológica le ha permitido desarrollar su capacidad de comunicarse con fluidez en su entorno y de trabajar en equipo, lo que supne un 19,2 \% del total. En el siguiente curso, el número de estudiantes coincide en esta respuesta (total 10 estudiantes), lo que equivale a un 15,4\% del total. En resumen, en estos dos años en que se ha registrado los datos, un total de 20 estudiantes (de 117) valoran que las clases teóricas, donde predomina la magistralidad, les ha aportado este aprendizaje, lo que equivale a un total de $17,1 \%$ (Ver Tabla 1 ). 
Tabla 1. Resultados de las respuestas obtenidas con las clases teóricas

\begin{tabular}{|c|c|c|}
\hline Curso & Respuestas afirmativas & Porcentajes \\
\hline $\mathbf{2 0 1 6 - 1 7}$ & 10 & 19,2 \\
\hline $\mathbf{2 0 1 7 - 1 8}$ & 10 & 15,4 \\
\hline Total & 20 & 17,1 \\
\hline
\end{tabular}

b) Con relación al trabajo grupal obligatorio, las respuestas obtenidas indican que, durante el curso 206-17, del total de estudiantes que respondieron la encuesta $(\mathrm{N}=52), 50$ afirman que éste les ha permitido desarrollar el aprendizaje de la capacidad que aquí se analiza, lo que supone un 96,2 \%. En el segundo año (2017-18) y con número de encuestas respondidas $\mathrm{N}=65$, son los/as 65 estudiantes los/as que realizan esta afirmación, lo que supone que el $100 \%$ de los/as mismos/as admiten haber desarrollado esta habilidad con el trabajo grupal. En conjunto, se tiene que 115 estudiantes responden positivamente, alcanzando un 99,1 \% (Ver Tabla 2).

Tabla 2. Resultados de las respuestas obtenidas con el trabajo grupal

\begin{tabular}{|c|c|c|}
\hline Curso & Respuestas afirmativas & Porcentajes \\
\hline $\mathbf{2 0 1 6 - 1 7}$ & 50 & 96,2 \\
\hline $\mathbf{2 0 1 7 - 1 8}$ & 65 & 100 \\
\hline Total & 115 & 99,1 \\
\hline
\end{tabular}

c) Con relación a las clases prácticas en el curso 2016-17, 41 estudiantes apuntan haber aprendido la habilidad con esta estrategia metodológica (78,8 \%). En el curso 2017-18, que responden afirmativamente 37 , suponiendo un $56,9 \%$. Se tiene que, en total, son 78 los/as estudiantes que admiten haberla aprendido, lo que supone un 66,7 \% (Ver Tabla 3).

Tabla 3. Resultados de las respuestas obtenidas con las clases prácticas

\begin{tabular}{|c|c|c|}
\hline Curso & Respuestas afirmativas & Porcentajes \\
\hline $\mathbf{2 0 1 6 - 1 7}$ & 41 & 78,8 \\
\hline $\mathbf{2 0 1 7 - 1 8}$ & 37 & 56,9 \\
\hline Total & 78 & 66,7 \\
\hline
\end{tabular}


¿Es posible que nuestros/as estudiantes adquieran la habilidad de comunicarse con fluidez en su entorno y de trabajar en equipo?

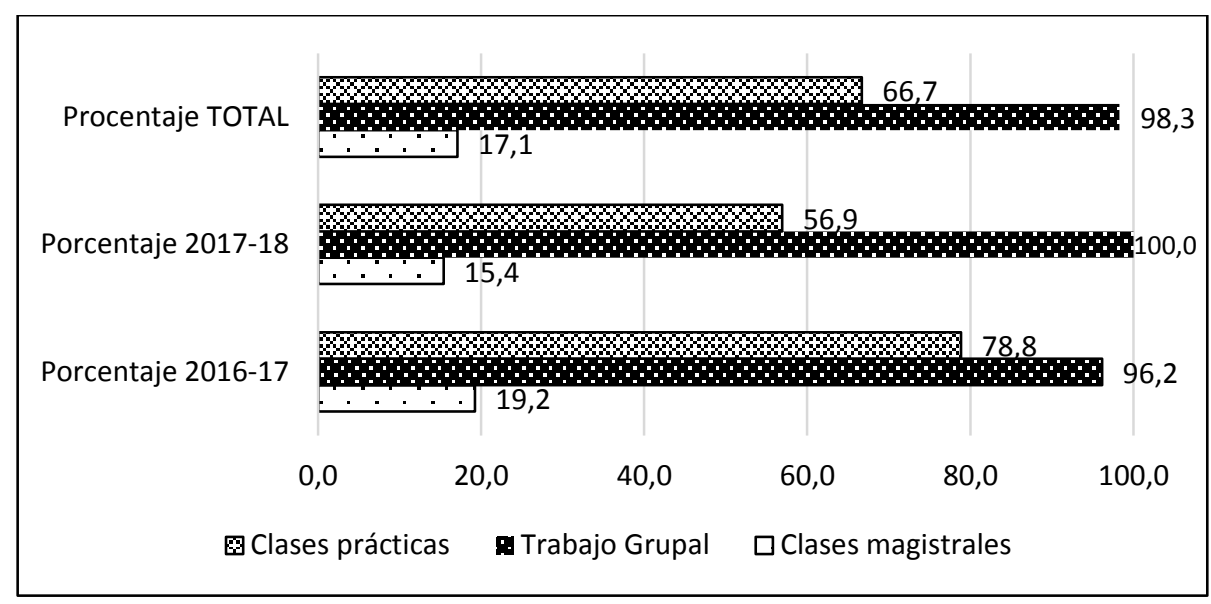

Fig. 1 Comparación porcentual de los resultados atendiendo a las estrategias metodológicas

\section{Análisis de los resultados}

Los datos obtenidos de la revisión de la literatura del currículum institucional revela que las tres competencias que aparecen en el Libro Blanco (comunicación oral y escrita en la lengua nativa, trabajo en equipo y el trabajo en equipo de carácter interdisciplinar) se reflejan en la competencias general que se contempla en el Título de Grado como capacidad de comunicarse con fluidez en su entorno y de trabajar en equipo. Además, señalar la relevancia que ostenta dicha competencia en el Título al estar contemplada en el ochenta por ciento de los módulos en los que se estructura el mismo. Y, respecto al Código Deontológico, decir que visibiliza la importancia de desarrollar esta capacidad en el ejercicio profesional, tanto en lo que al servicio a la población refiere, como en la gestión de la propia profesión.

Atendiendo a los resultados presentados en el apartado ¿Qué dicen los/as estudiantes sobre la adquisición de la habilidad como resultado de la experiencia docente?, se desprende que la estrategia metodológica que cuenta con más tiempo de actividad en el plan de estudio y, por tanto, en el proyecto docente, como son las clases teóricas con el gran grupo, cuya finalidad principal es la de presentar y tratar los contenidos conceptuales de la materia, es la que menos impacto tiene sobre el aprendizaje de la habilidad capacidad de comunicarse con fluidez en su entorno y de trabajar en equipo. En segundo lugar, se encuentran las clases prácticas, cuya finalidad es tratar de reforzar y/o complementar los contenidos a través de actividades en las que los/as estudiantes adoptan un papel más activo. En tercer, y último lugar, destaca de manera importante los resultados obtenidos respecto al aprendizaje 
que aporta el trabajo grupal obligatorio, de manera que, la estrategia de trabajo que lo define favorece que los/as estudiantes adquieran y/o desarrollen esta habilidad.

\section{Conclusiones}

De todo lo anterior, se desprende que la competencia general capacidad de comunicarse con fluidez en su entorno y de trabajar en equipo, es una competencia que ostenta un notable protagonismo en la formación de los/as trabajadores/as sociales, a la vez que en su práctica profesional $\mathrm{y}$, por tanto, se trata de una competencia sobre la que la actuación docente ha de ser eficaz.

Con relación dar respuesta a si es posible que nuestros/as estudiantes adquieran la habilidad de comunicarse con fluidez en su entorno y de trabajar en equipo, y atendiendo a los datos aportados por los/as estudiantes, se puede afirmar que sí pueden adquirirla, pero señalar que requiere de estrategias prácticas, en las que estos/as pongan en escena sus capacidades para identificarlas y modelarlas, pues las clases de carácter tradicional, tal y como se sigue configurando las clases teóricas del gran grupo, difícilmente supone un impacto en el desarrollo de los/as estudiantes.

Decir también que, por la valoración que expresan los/as estudiantes, se puede presagiar que la experiencia de carácter constructivista seguida con el trabajo grupal basada en teorías pedagógicas (que esencialmente contemplan al alumnado como agente que aprende a partir de su contexto/realidad sociocultural, a partir de los conocimientos que ya posee, y que experimenta atendiendo a los significados que la vivencia de aprendizaje le aporta al conectar con sus intereses, a la par que ser actores del descubrimiento de los aprendizajes) son teorías que favorecen de manera muy acertada la consecución de habilidades como la que se ha analizado en este trabajo. Ello verifica que el esfuerzo de la innovación educativa que se viene impulsando, está siendo eficaz.

Para concluir, ante los resultados presentados que responden a la experiencia concreta de dos años en esta asignatura, se propone seguir avanzando en el proceso de análisis-reflexión sobre la tarea docente, profundizando en la misma. En este sentido, la actividad como servicio al alumnado, debe seguir madurando como acción del aprendizaje del profesorado, con lo que se propone que la acción nutre a la teorización de la práctica docente, a través de un aprendizaje (los/as docentes están aprendiendo)-servicio (a través de la realización de un servicio a su alumnado). 
¿Es posible que nuestros/as estudiantes adquieran la habilidad de comunicarse con fluidez en su entorno y de trabajar en equipo?

\section{Referencias}

Agencia Nacional de Evaluación de la Calidad y Acreditación (2004). Libro Blanco Título de Grado de Trabajo Social. Recuperado de http://www.aneca.es/var/media/150376/libroblanco_trbjsocial_def.pdf

Aramburuzabala, P. (2013). Aprendizaje-Servicio: Una herramienta para educar desde y para la justicia social. Revista internacional de educación para la justicia social, vol. 2, Núm. 2, p. 5-11. Recuperado de http://www.rinace.net/riejs/numeros/vol2num2/editorial.pdf

Boni, A., López, E. y Barahona, R. (2013). Approaching quality of global education practices through action research. A non-governmental development organizationuniversity collaborative experience. International Journal of Development Education and Global Learning 5(2), pp. 31-46.

Cano-Ramírez, A. (2014). Exploración de las prácticas docentes con enfoque de Educación para el Desarrollo para la Ciudadanía Global. Aproximación diagnóstica en los títulos de grado de las universidades españolas tras la implementación del EEES. Universidad de Las Palmas de Gran Canaria, España. Recuperado de http://hdl.handle.net/10553/12352.

Consejo General del Trabajo Social (2012). Código Deontológico de Trabajo Social. Recuperado de http://www.comtrabajosocial.com/documentos.asp?id=1317

Díaz Pinto, C.F. (2010). Viejas y Nuevas Ideas en Educación. Una historia de la pedagogía. Madrid: Editorial Popular.

Naciones Unidas (2015). Resolución aprobada por la Asamblea General de 25 de septiembre de 2015: 70/1 Transformar nuestro mundo: la Agenda 2030 para el

Desarrollo Sostenible. Recuperado de

http://www.un.org/es/comun/docs/?symbol=A/RES/70/1

Ortega Carpio, Mª L. (2007). Estrategia de Educación para el Desarrollo de la Cooperación Española. Madrid: Agencia Española de Cooperación Internacional al Desarrollo (Ministerio de Asuntos Exteriores y Cooperación). Recuperado de http://www.aecid.es/Centro-

Documentacion/Documentos/Planificación\%20estratégica\%20por\%20sectores/estrategi a_educacion_desarr.pdf

Universidad de Las Palmas de Gran Canaria. Proyecto de Título de Grado de Trabajo Social de la Universidad de Las Palmas de Gran Canaria 2010-11. Recuperado de www.ulpgc.es 